\title{
Exploratory Outcome Measure
}

National Cancer Institute

\section{Source}

National Cancer Institute. Exploratory Outcome Measure. NCI Thesaurus. Code 198724.

Exploratory measures that are used to evaluate and/or form hypotheses about the intervention outcome(s). 